the course of the fighters so as to bring about interception of the bombers. Bomber control may be carried out if required by a controller at another plan position indicator.

An interesting adjunct to the equipment is the simulation of electronic radar counter-measures both by parachuted noise jammers and by the dropping of strips of metal foil ('window'). In both cases means are provided in the equipment to take into account the effect of the prevailing wind on the drift of the jammers.
In the design of this versatile installation, the likelihood of future changes in the performance of aircraft and radar, and of air defence procedure in general, have been borne in mind, and the necessary modifications and additions to the installation to deal with such changes are readily made.

Equipment similar in principle to that demonstrated may be used for simulating marine exercises in which both radar and asdic are used, and it may also be used for training in civil air-traffic controls. A. F. Wilkins

\title{
CHEMISTRY AND QUANTUM CHEMISTRY
}

$\mathrm{I}^{\mathrm{N}}$ the course of the past twenty-five years the language of quantum mechanies has invaded almost every branch of chemistry. Often this has been at a very qualitative level involving only such basic concepts as those of stationary states and energy-levels. Many of the most useful ideas, however, are ones which do not belong to accurate quantum mechanics but arose first in certain approximate calculations and have since been developed by using them to explain experimental facts. These range from the idea of a crystal potential which is used to make qualitative predictions about molecular complexes, to ideas such as hybridization and resonance which are used qualitatively to describe the structure and reactivities of molecules. Unfortunately, it is becoming very clear that most of these ideas need careful re-examination both to establish their quantum mechanical status and to show how they can be refined or modified to explain the more extensive and more accurate experimental results now avail able.

It was very appropriate, therefore, that this problem of the correspondence between the concepts of chemistry and those of quantum chemistry should have been the subject of a panel meeting held at Vălådalen, in the Swodish mountains, during August $26-30$ and organized by Dr. P. O. Löwdin, director of the Quantum Chemistry Group in Uppsala. The audience consisted primarily of experimentalists who had spent the previous month there at the summer school on quantum chemistry, and the panel included both chemists and quantum chemists. The meetings were pleasantly informal, and, since the number of prepared contributions was deliberately kept small, there was ample time to discuss fundamental problems.

The theory of molecular structure based on the overlapping of hybrid atomic orbitals which are concentrated in the bond directions was one topic discussed, and in particular the idea of assigning a constant atomic radius to each kind of hybrid. Experimental methods of determining molecular structure using micro-waves, electron diffraction and X-rays were reviewed by Prof. B. Bak (Copenhagen), Prof. K. Hedberg (Oregon), Prof. O. Bastiansen (Trondheim) and Dr. I. Lindqvist (Uppsala), and they reported that the accuracy and flexibility of these methods had recently increased considerably. The results were shown by Prof. Bak and Dr. I. Hjalmars (Stockholm) to imply that the approximation of a constant radius for each hybrid is often accurate to $0.001 \mathrm{~A}$. Furthermore, as Prof. L. Pauling (Pasadena) showed, the rotational barriers can be predicted by including in the hybrids small amounts of higher angular terms, and the experimental confirmation of these predictions was discussed by Prof. L. Pierce (Notre Dame). On the other hand, Dr. Löwdin pointed out that the principle of maximum overlapping is quantitatively inaccurate even on an approximate theory and that the accurate theory does not even give any unique hybrids. In consequence, it is difficult to believe that the hybrids have any physical reality. Prof. R. S. Mulliken (Chicago) agreed that there is still no basic theoretical reason why covalent radii should be so constant.

There was general agreement on the artificial nature of the distinction often made between ionic and covalent binding. Both Dr. S. Lundqvist (Uppsala) and Prof. H. Shull (Indiana) pointed out that ionic wave-functions can sometimes give covalent properties and be almost identical with corresponding covalent wave-functions. This led naturally to a discussion of the method of 'atoms in molecules' which uses such wave-functions. Dr. R. Pauncz (Haifa) showed that, when the method is applied literally to hydrogen, the results are rather poor, but Dr. H. Preuss (Göttingen) defended the method in a modified form. It seems clear that the unmodified free atoms or ions do not provide the best units for describing the compound.

The concept of hyperconjugation is also in some difficulties. This type of delocalization of the electrons plays an important part in the excited states and ionized states of many molecules and in transition complexes, but it is not so clear that it is important in molecular ground-states. The concept was strongly defended by Prof. Mulliken, and he claimed that there is sufficient experimental evidence to substantiate it even for the ground-state. The concept of conjugation also has recently been attacked, but Prof. Mulliken thought this was because the simple Hückel theory sometimes exaggerated its effects, for example, in butadiene, whereas more recent theories give much better agreement with experiment. Dr. R. MeWeeny (Keele) argued that the study of diamagnetic susceptibility and proton magnetic resonance gives very strong evidence for conjugation, although Prof. Bak and Mr. R. A. Hoffman (Uppsala) pointed out that some proton magnetic resonance effects in conjugated molecules are not yet understood. Dr. S. Sunner (Lund) talked about difficultios in understanding resonance and strain energies and suggested that these are often due to the use of unrelated or inappropriate reference states.

A point which emerged clearly from the discussion is the growing tension between the so-called semi- 
empirical methods and those based on a priori calculations. Mr. C. Schäffer (Copenhagen), for example, described the successful use of crystal-field theory in explaining the spectra and properties of molecular complexes. Yet, as Dr. Löwdin insisted, the model does break down when refined too far and the empirical values of the parameters do not agree with those calculated. The resonance theory is another example of a theory which is still useful qualitatively and semi-quantitatively despite the fact that some of its assumptions are in serious error. The success of the simple Hückel theory, according to Dr. G. G. Hall (London), is not due to the accuracy of its assumptions but to the mathematical resemblance between its equations and those of a more accurate theory, and it may be that such resemblances occur for other theories.

The urgent need for more accurate theories and concepts appropriate to them was felt several times during the meeting. Dr. H. G. Bennewitz and Dr. G. Gräff (Bonn) underlined it by describing molecularbeam experiments which yield very accurate values of many molocular constants. Prof. F. A. Matsen (Texas) and Dr. Hall favoured the method of different orbitals for different spins as a method more accurate than that of molecular orbitals yet as easy to visualize. There was also a vigorous discussion on the rival merits of natural orbitals and population analysis as methods of understanding complicated wave-functions.

A discussion of the future of quantum chemistry provoked some differences of opinion. In the view of Prof. Mulliken, the calculation of good molecular orbital wave-functions, which is now beginning, will help greatly to clarify molecular structure and will enable generalizations about correlation energies to be made. Dr. Löwdin thought that the effects of correlation and relativistic corrections will be of importance in many chemical problems and should be investigated using more accurate wave-functions. On the other hand, Dr. Hall believed that a priori calculations will never be possible for more than a small number of molecules and that it is just as important to study accurately the relations between the molecules of a molecular series. Prof. Matsen considered that the experimentalists' need for concepts with which to organize and understand their results is so urgent that they may be forced to invent these without waiting for the development of quantum mechanical ones. It was Prof. Pauling's hope that the present introspective phase of quantum chemistry, though obviously necessary, will not last very long, since there are many urgent chemieal problems which could be solved more easily theoretically than experimentally.
G. G. HALL

\section{NATURAL SELECTION IN MAN}

TN a year marked by the celebration of the Darwinian-Wallace centenary, it is fitting that a symposium, sponsored by the Society for the Study of Human Biology and held on November 8 in the Department of Anatomy, University College, London, should have focused attention on modern analyses of the action of natural selection in human populations. The symposium comprised six papers, and in five of these attention was directed to a greater or lesser extent towards genetical matters, knowledge of which is basic to studies of the promotion of evolutionary change by selective forces. In the final contribution, the field was still further expanded by the consideration of a number of broader questions connected with primate (including human) evolution.

The balance of the whole symposium was such as to indicate clearly the extent to which, despite much intensive work, knowledge of many aspects of human inheritance and selection theory still falls short of that now available for some other animal species. Little is known, for example, of the multifactorial control of quantitative human features; but, on the other hand, a considerable body of information has accumulated in relation to certain groups of major human genes, and several speakers addressed themselves to problems associated with the action of selection upon individuals distinguished by the possession of easily identifiable allelomorphs.

Two of several problems basic to present-day studies of natural selection in man are, as pointed out by Prof. L. S. Penrose (University College, London) in an opening paper, the recognition of these major genetic factors and the determination of the frequencies at which they exist in different populations. The first of these aims has, in many cases, now been attained by analyses of the human pedi- grees to the compilation of which much early work was directed. The second has only been achieved on any considerable scale in the case of such factors as those which determine the blood groups, but in this particular case, data are now available from widely scattered regions of the world.

Genetic factors of this type must, as pointed out in the opening paper, have arisen at some time by mutation, but such origins of new variability are rarely recorded. Thus, despite the thoroughness with which the blood-group systems have boen studied, mutation in them has not been observed. Indirect evidence, however, indicates its occurrence, sometimes at relatively high rates. Achondroplasia, for example, results from the effect of a single dominant major gene which has in certain populations remained in circulation for long periods. The condition is associated with a marked degree of infertility, and the genetic wastage which thereby results must pre sumably be made good continually by new mutation.

Recessive genes which, when substituted in double dose into certain gonetic complexes, may exert markedly deleterious effects on viability exist in heterozygous form in all populations. They provide a source of potential variability which if liberated would, even in the absence of mutation, form an adequate basis for considerable amounts of evolutionary change. The scope and selective significance of this potential variability are difficult to assess from the available human data, but a reminder of their extent was given by Prof. Th. Dobzhansky, who, as the guest speaker, presented in outline the results of his own extensive work on the hidden genetic variability in wild populations of Drosophila. 